\title{
Neuer Baustein für die antidepressive Therapie?
}

Fragestellung: Ist eine psychologische, internetbasierte Intervention bei Probanden mit leichten bis mittelschweren depressiven Symptomen der üblichen Behandlungsform überlegen?

Hintergrund: Internetbasierte Interventionsangebote bei Patienten mit depressiver Symptomatik sind im Fokus der klinischen Forschung. Man unterscheidet zwischen Angeboten, die von Probanden selbstständig durchgeführt oder durch Kliniker begleitet werden. Bisher konnte zwar gezeigt werden, dass sich internetbasierte Interventionen positiv auf Probanden mit depressiver Symptomatik auswirken, jedoch war die statistische Aussagekraft bei den allermeisten Vorstudien sehr gering, beziehungsweise es existieren einige Vorstudien, die keinen Benefit durch solche Maßnahmen zeigten.

Patienten und Methodik: Die randomisierte, kontrollierte, einfach verblindete klinische Studie richtete sich an Probanden mit leichten bis mittelschweren depressiven Symptomen (Patient Health Questionnaire-9, PHQ-9). Weder die vorhandene Diagnose einer depressiven Störung noch eine vorbestehende Behandlung waren Einschlusskriterien für diese Studie. Knapp über 2.000 Probanden im Alter von 18 bis 65 Jahren wurden interviewt. Letztendlich wur-

Klein JP, Berger T, Schröder J et al. Effects of a psychological internet intervention in the treatment of mild to moderate depressive symptoms: Results of the EVIDENT Study, a randomized controlled trial. Psychother Psychosom 2016; 85: 218-28 den 1.013 in die Studie eingeschlossen und in die zwei Studienarme Internet intervention $(\mathrm{n}=509)$ und Care as usual $(\mathrm{n}=504)$ randomisiert. Im Interventionsarm erhielten die Probanden ein zwölfwöchiges verhaltensthera- peutisches Online-Programm (Deprexis ${ }^{\circledast}$ ). Bestandteile des interaktiven Programms aus zehn Modulen sind Audioaufnahmen, Arbeitsblätter, Zusammenfassungen und knappe Tagesnachrichten, zugestellt mittels SMS oder E-Mail. Im Kontrollarm wurde durch die klinischen Prüfer in keiner Form Einfluss auf die aktuelle Behandlung genommen. Primärer Studienendpunkt war der PHQ-9 Wert nach drei Monaten (nach der Intervention) und nach sechs Monaten (Follow-up). Sekundäre Endpunkte waren die Hamilton Depression Rating Scale (HAMD24), das Quick Inventory of Depressive Symptomatology, das Mini International Neuropsychiatric Interview und ein Fragebogen zur psychischen und allgemeinen Lebensqualität.

Ergebnisse: Es zeigte sich nach drei Monaten eine statistisch signifikant höhere PHQ-9-Verbesserung im Interventionsarm im Vergleich zum Kontrollarm. Die Effektstärke betrug d = 0,39. Die Ergebnisse blieben auch bis zum Zeitpunkt der Follow-upUntersuchung stabil. Symptomfragebögen mit Fremd-Rating wiesen geringere Effektstärken als Fragebögen mit Selbst-Rating auf (PHQ-9: $\mathrm{d}=0,39$ vs. HAMD-24: $\mathrm{d}=0,19$ ). Mindestens eine minimale, klinisch relevante Verbesserung des PHQ-9-Ergebnisses erreichten 35,6\% der Patienten im Interventions- und 20,2 \% im Kontrollarm. Die Number needed to treat betrug 7.

Schlussfolgerungen: Die Autoren folgern, dass die internetbasierte Intervention einem üblichen Behandlungsschema in Bezug auf die Reduktion von leichten bis mittelschweren depressiven Symptomen überlegen ist. Das Ausmaß dieser Ergebnisse ist ihrer Ansicht nach klinisch bedeutend, besitzt Relevanz für das Gesundheitssystem und sollte zum Standardrepertoire der Depressionsbehandlung hinzugefügt werden.

\section{- Kommentar von David Herzog, Mainz}

\section{Für eine generelle Therapieempfehlung ist es noch zu früh}

Die vorliegende randomisierte, kontrollierte, einfach verblindete, prospektive, multizentrische Studie glänzt mit guter Studienqualität, einer großen Fallzahl und einem durchdachten naturalistischen Studiendesign, unabhängig von kategorialen Diagnosen. Kritisch zu sehen sind die recht geringen Raten an erfolgreich abgeschlossenem Post-Assessment (21,6\%) und Follow-up-Assessment (24,6\%), was belastbare Aussagen zu diesen beiden Parametern erschwert. Außerdem konnte aufgrund des Studiendesigns keine doppelte Verblindung durchgeführt werden, was die Aussagekraft der Ergebnisse schmälert. Dies ist vor allem deshalb relevant, da die ermittelten Effektstärken eher im niedrigen Bereich anzusiedeln sind und daher eigentlich eine möglichst hohe Studienqualität nötig ist, um probate Aussagen tätigen zu können. In Anbetracht verschiedener Vorstudien mit zum Teil konträren oder unzureichend statistisch untermauerten Ergebnissen bringt die vorliegende Studie einen großen Zugewinn. Hieraus eine ge- nerelle Therapieempfehlung für Patienten mit leichten bis mittelschweren depressiven Symptomen - wie die Autoren der Studie dies tun - abzuleiten, wäre allerdings zu früh, denn eine weitergehende intensive Beschäftigung mit diesem Thema, inklusive einer Kosten-Nutzen-Analyse, ist erforderlich.

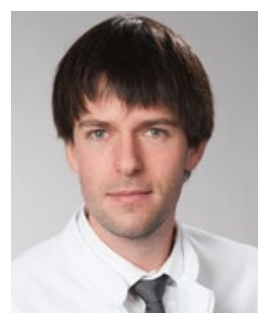

Dr. med. David Herzog, Mainz

Klinik für Psychiatrie und Psychotherapie, Universitätsmedizin Mainz E-Mail: david.herzog@unimedizin-mainz.de 